\title{
Does transient blocking of axonal conduction occur in uraemic neuropathy?
}

\author{
D. W. M E Y E R, J . - P. H A L O N E N, A. H . L A N G, \\ V. K U USE L A, A N D J . FOR S T R Ö M \\ From the Department of Clinical Neurophysiology, University Central Hospital, Turku, Finland
}

SUMMARY In uraemic and other patients with neuropathies, motor unit spike intervals were analysed using a special computer program to detect prolonged intervals of twice the normal duration, so-called "double intervals." These may have different causes-trigger failure, double discharge, or a transient fall in the firing rate of coactive motor units. There was not sufficient evidence that transient conduction blockings will occur in the main axon in uraemic neuropathies.

The pathogenetic mechanism underlying uraemic neuropathy has been widely discussed (Asbury, 1975). In earlier studies of histopathological changes in uraemic patients, a primary demyelination of peripheral nerve fibres was said to be typical of this neuropathy (Dayan et al., 1970; Dinn and Crane, 1970). Nonetheless, more recent studies state that demyelination is secondary to axonal degeneration (Dyck et al., 1971; Thomas et al., 1971; Gilliatt, 1973). Nielsen (1974) points out, however, that the latter view is mostly based on studies of advanced neuropathy in terminal states of uraemia.

Maximal nerve conduction velocity (NCV) decreases even in early states of uraemia, the dysfunction of peripheral nerves often being subclinical (Preswick and Jeremy, 1964; Honet et al., 1966; Kemple, 1967; Jebsen and Tenckhoff, 1969; Nielsen, 1973). A minor decrease of NCV may, however, be a consequence of primary axonal degeneration or minor demyelination of nerve fibres (Gilliatt, 1966; Buchthal, 1973; Kaeser, 1975). Moreover even non-structural dysfunction of the axonal membrane may be responsible for reversible reduced NCV in uraemia (Thomas, 1971; Nielsen, 1974; Lang and Forsström, 1977). Thus both morphological and NCV studies have furnished contradictory information about the pathogenetic mechanism underlying uraemic neuropathy.

This work was supported by the National Research Council for Medical Sciences, Finland.

Address for reprint requests: Detlef W. Meyer, Neurologische Klinik, Moorenstr. 5, D-4000 Düsseldorf, Federal Republic of Germany.

Accepted 25 January 1978
In their studies on the firing pattern of single motor units, Dietz and Freund $(1974,1975)$ described systematic differences in the statistical distribution of motor unit potential (MUP) firing intervals in different polyneuropathies. In cases of alcoholic polyneuropathy, which is probably due to primary axonal degeneration (Asbury, 1975), the interval histogram seemed to be evenly distributed, as is the case with normal control subjects. In cases of uraemic neuropathy, however, a second clustering of intervals seemed to occur. These intervals were about twice longer in duration than the mean interval duration. This dichotomy was best recognised when using the joint histogram technique, which seems to suggest that the prolonged intervals are generally located between two intervals of normal duration.

According to Dietz and Freund (1974, 1975), the occasional occurrence of single long MUP intervals is caused by a transient conduction block in the main motor axon. Transient conduction blocks have been shown to occur in connection with experimental demyelination in both central (McDonald and Sears, 1970) and peripheral (Rasminsky and Sears, 1972) nerve fibres. By analogy, Dietz and Freund $(1974,1975)$ concluded that the dichotomous joint interval histogram of MUP intervals indicates segmental demyelination in uraemic neuropathy.

If the findings established by Dietz and Freund (1974, 1975) of a specific firing pattern of motor units in uraemia and their interpretation of this phenomenon are correct, research of the motor unit firing pattern would not only elucidate the pathogenetic mechanism of different neuropathies, 
but could also be employed to differentiate between axonal degenerative and demyelinating neuropathies. Instead of adapting the joint interval analysis method used by Dietz and Freund (1974, 1975), we have made a computer program for the identification of single MUP intervals whose duration is about twice the prevailing intervals. We call this the double interval (DI) firing pattern, this term, however, being purely descriptive with no inferences being drawn on the mechanism of its occurrence. After the identification of the DI firing pattern we subsequently checked on what really occurs in the EMG at that time from our tape records. A preliminary report of these results has been given by Meyer and Lang (1977).

\section{Subjects and methods}

There were three groups of subjects: (1) 19 uraemic patients, 13 attending maintenance haemodialysis and showing different degrees of peripheral nerve dysfunction (clinical symptoms and signs, and lowered sensory NCV), and six receiving conservative therapy only; (2) 22 patients with either non-uraemic polyneuropathies of different origin or radicular neuropathies; (3) 28 control subjects.

We made 227 motor unit records, and over 70000 spike intervals were analysed systematically.

The EMG was recorded mainly from the first dorsal interosseous and tibialis anterior muscles. In the normal group a smaller collection was made from the extensor indicis proprius muscle. Concentric EMG electrodes (DISA 9013L0501 with an external diameter of $0.45 \mathrm{~mm}$ ) and commercial EMG equipment (DISA 14A30) were used. In a later series of recordings a second EMG electrode was inserted in the vicinity of the first, the recordings being made by using two channels.

Patients were instructed to activate a single motor unit (MU) or an MU with a prominent positive or negative spike component. As the subject learned to keep the MU firing regularly by audiovisual feedback (Basmajian, 1963), the EMG activities from this and the second electrode were recorded on an FM tape recorder.

For interval analysis the EMG activity from the first channel of the recorder was fed into a non-commercial peak-detector with adjustable and monitorable amplitude limits. The detector changed the EMG spikes falling within the limits of the trigger window into pulses that triggered a multichannel computer (DIDAC 4000) working as an interval counter. As a rule, a time resolution of $0.1 \mathrm{~ms}$ was employed. After the successive spike intervals had been counted (usually 200-
400 per single MU), values and identification numbers were printed out and stored separately on a punch tape. The information on punch tape was then analysed using a computer program which was specifically designed to identify single DIs. The statistical program was set up by one of the team (VK).

\section{COMPUTER PROGRAM FOR IDENTIFICATION OF DI} FIRING PATTERN

The program used for the identification of single DIs contained two steps. Firstly, it calculated the mean and the variance of the interval series and, adapting rather liberal statistical criteria, identified all "possible DIs"- that is, all intervals having a duration of about twice the mean. Secondly, the program halved the value of each "possible DI" and compared this value with the preceding and following interval sequence. The sequence length could be adjusted to twice 3,4 , or 5 intervals. The program then calculatec the probability that each halved "possible DI" belonged to this interval sequence, and printed the sequence number, value, and probability of each DI fulfilling the predetermined probability criterion. The optimal probability value of 0.5 showed that the interval was exactly twice the mean interval of a potential sequence of an otherwise stable firing rate. The DI probability (DIprob) deviated from this value the more that irregularities occurred in the firing rate during the MUP sequence, and the shorter or longer halved intervals were compared with the mean interval. Short DIs gave DIprob values below 0.5 , long DIs gave values above 0.5 .

On the basis of their values and identification numbers the corresponding spike intervals could later be identified in the primary tape records. Controls of the original EMG records were made from a sk $\rightarrow$ rt run of the EMG record showing the identified DI firing pattern. Most of the material was controlled by the multichannel analyser functioning as a memory oscilloscope. Further analysis of 52 consecutive identified DIs (derived from seven uraemic patients and one patient with a radicular syndrome) was made by using ultraviolet recordings (Photocorder, Type 9975). The extended time and amplitude scale of the recorder made very detailed analysis of the primary record possible.

\section{Results}

ASSESSMENT OF DI FIRING PATTERN

Figure 1 shows three MUP series recorded from the anterior tibial muscle of a uraemic patient. A single, long MUP interval occurs centrally in each 


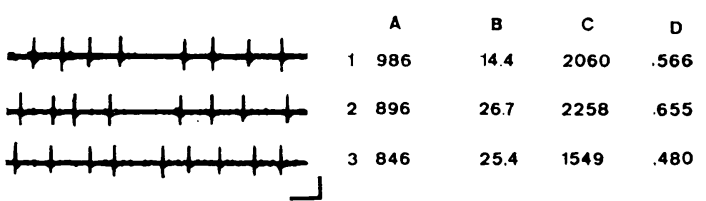

Fig. 1 Three traces with double interval firing pattern. Numbers on right show the information given by the computer program from each trace. A: mean interval duration of five intervals before and after the identified double interval (time resolution $0.1 \mathrm{~ms}$, eg in $1 \mathrm{~A}: 986=98.6 \mathrm{~ms}) ; B: S D$ of the interval sequence ( $m s$ ); $C$ : duration of the identified double interval (time resolution $0.1 \mathrm{~ms}$ ); D: DIprob (see Methods). Calibration $1 \mathrm{mV}$ and $100 \mathrm{~ms}$.

trace. The value for one half of the length is longer in the first two traces and shorter than the mean duration of the flanking intervals in the third trace. Consequently the computed DIprob (see Methods) is above 0.5 in the first two cases, and below 0.5 in the third case. Furthermore, in the first case the motor unit fires quite regularly before and after the single long interval, the duration of which is nearly twice the mean of the flanking intervals. In the first example DIprob, therefore, differs less from the optimal value 0.5 than in the second.

From the principle of computing DIprob it follows $a$ priori that (1) even one single DI is sufficient to reveal a DI firing pattern of a given motor unit, and (2) that in the case of very irregular firing the program may be insufficient to recognise that single motor units are deficienteven should this happen biologically.

To ensure the proper working of the DI firing pattern detection program, we recorded the motor unit activities from our own muscles. The limits for DIprob were set to $0.3-0.7$ in the pilot experiments. By using these statistical limits it was im- possible to produce DI firing pattern voluntarily by activating the motor units in an irregular wayshort muscle twitches, slow increase and decrease in the firing frequency, and activation of the motor unit with the lowest possible repetition rate, thus causing a very irregular firing frequency.

\section{INCIDENCE OF DI FIRING PATTERN}

The Table shows the results of systematic DI firing pattern analysis. In the control group the incidence of DI firing pattern of the analysed intervals was nearly identical in the two proximal muscles, but significantly higher in the distal one. In the non-uraemic patient group the incidence of DI firing pattern per intervals was significantly higher in the tibialis anterior, but lower in the first dorsal interosseous muscle (this difference possibly being due to the small number of recordings from the latter muscle). In the case of the uraemic patients the incidence of DI firing pattern was highest regardless of the muscle. The patients under maintenance haemodialysis showed somewhat higher incidence figures compared to those attending conservative therapy.

The incidence of DI firing pattern per motor units did not show such clear differences between the various groups. The occurrence of DI firing patterns and the increase in their number appeared to be confined to a limited number of motor units, while the rest displayed normal firing patterns in this respect, even in uraemia.

\section{CHECKING DI FIRING PATTERN}

Even a superficial examination of the records from the small oscilloscope of our multichannel analyser confirmed that about one-third of the DI firing patterns was due to occasional triggering failure. On the basis of careful examination of ultraviolet recordings giving very detailed information of the EMG activity, 52 consecutive DI firing patterns

Table Incidence of double interval firing in patients and control subjects

\begin{tabular}{|c|c|c|c|c|c|c|c|}
\hline & \multirow{2}{*}{\multicolumn{2}{|c|}{ Control subjects }} & & \multicolumn{4}{|l|}{ Patients } \\
\hline & & & & \multicolumn{2}{|c|}{ Non-uraemic neuropathy } & \multicolumn{2}{|c|}{ Uraemic neuropathy } \\
\hline Number of individuals & 28 & & & 22 & & $\begin{array}{l}19 \\
(13)^{*}\end{array}$ & \\
\hline $\begin{array}{l}\text { Muscles } \\
\text { Number of motor units analysed } \\
\text { Number of motor units showing }\end{array}$ & ID $_{26}$ & $\begin{array}{l}\text { EIP } \\
14\end{array}$ & ${ }_{23}^{T A}$ & ${ }_{13}^{\text {ID }}$ & ${ }^{T A}$ & ${ }_{24}^{\text {ID }}$ & ${ }^{T A}{ }_{67}$ \\
\hline $\begin{array}{l}\text { DI firing patterns } \\
\text { Number of intervals analysed } \\
\text { Number of DI firing patterns } \\
\text { Number of DI firing patterns }\end{array}$ & $\begin{array}{r}13 \\
8188 \\
15\end{array}$ & $\begin{array}{r}4 \\
5000 \\
5\end{array}$ & $\begin{array}{r}9 \\
14538 \\
13\end{array}$ & $\begin{array}{r}1 \\
3851 \\
1\end{array}$ & $\begin{array}{r}30 \\
14683 \\
33\end{array}$ & $\begin{array}{r}12 \\
6989 \\
24\end{array}$ & $\begin{array}{r}34 \\
17225 \\
81\end{array}$ \\
\hline per 1000 intervals analysed & 1.83 & 1.00 & 0.89 & 0.26 & 2.24 & $\begin{array}{l}3.43 \\
(5.15)^{*}\end{array}$ & $\begin{array}{l}4.76 \\
(4.27)^{*}\end{array}$ \\
\hline
\end{tabular}

* Patients attending maintenance haemodialysis.

ID = first dorsal interosseous; $E I P=$ extensor indicis proprius; $T A=$ tibialis anterior 
were broken down into three different categories.

1. In 23 cases the DI was clearly caused by a triggering failure. In most cases this was brought about by a second MUP derived from a coactive motor unit, interfering occasionally with the larger MUP under study. Their interference resulted in a small compound action potential, the amplitude of which remained below the triggering level (Fig. 2).

2. In seven cases intervals meeting DI criteria were preceded by a double discharge of the motor unit (Fig. 3). All these DIs were found in the patient suffering from a radicular syndrome.

3. In 22 cases no triggering failure and no double discharge could be detected. A second motor unit was firing repetitively at the moment when the first motor unit showed DI firing pattern in 14 of these cases. It is apparent from Fig. 4 that the firing rates of both motor units fall simultaneously. Figure 5 summarises the cases in this category. A minor increase in the firing rate of the coactive motor unit during DI firing pattern only occurred in one case, the rest showing a simultaneous fall of activity. This simultaneous inhibition of activity seemed in many cases to be restricted to the muscle territory under study, but sometimes is extended to a larger part of the muscle (Fig. 4).

In the remaining cases in this category the motor unit showing the DI firing pattern was the only active motor unit to be identified in the record derived from that muscle territory.

GENERAL FIRING PATTERN OF MOTOR UNITS IN URAEMIC PATIENTS

The firing rate of motor units was, on average, identical in uraemic patients and in control subjects (Fig. 6). A gradual decrease in frequency and an increase in variance was, however, often recognised in uraemic patients compared with the relatively stable firing pattern in controls even during very long recordings. Many uraemic patients had small involuntary muscle twitches

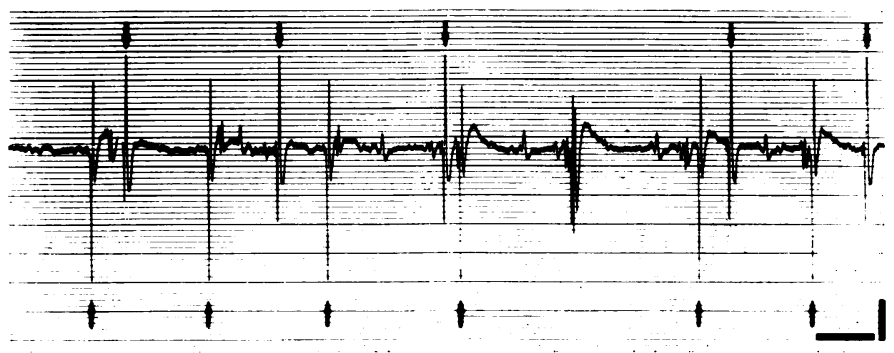

Fig. 2 Interference of two different motor unit potentials (upper and lower arrows respectively). Note marked decrease in amplitude during interference. Calibration $200 \mu \mathrm{V}$ and $50 \mathrm{~ms}$.

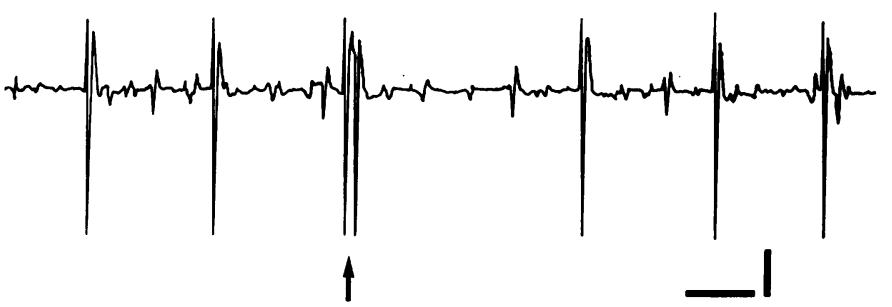

Fig. 3 A prolonged interval after double discharge. The double discharge is indicated by arrow. Calibration $200 \mu \mathrm{V}$ and $50 \mathrm{~ms}$.

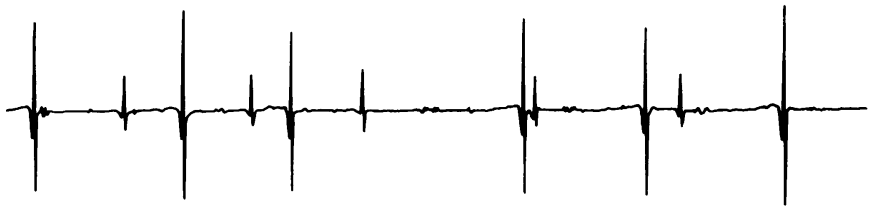

Fig. 4 Synchronous decrease of firing rate in different motor units. Lower trace is recorded with another needle electrode in the vicinity of the upper trace, and the activity also shows attenuation during

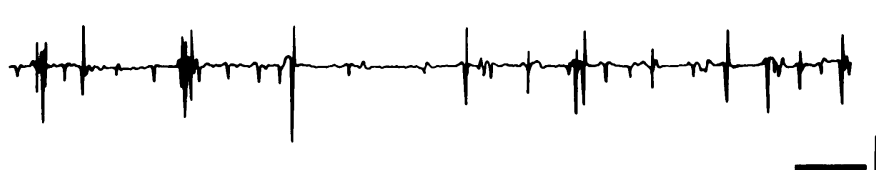
double interval firing pattern. Calibration $500 \mu \mathrm{V}$ and $50 \mathrm{~ms}$. 


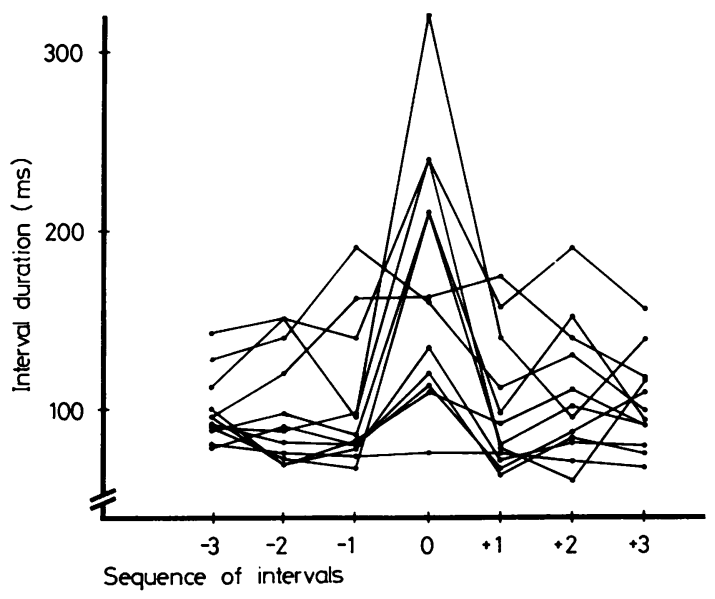

Fig. 5 Change of firing rate in coactive motor units during double interval firing pattern. Interval number zero shows duration of the first interval starting after the beginning of the identified double interval.

even at the start of a tonic isometric contraction. Later during the contraction new motor units showing phasic firing pattern were recruited.

No distinct correlation could be demonstrated between the firing rate and the incidence of DI firing pattern (Fig. 6). If anything, the DI firing patterns tended to be more common in cases with a relatively high $\mathrm{MU}$ firing rate.

\section{Discussion}

The results obtained indicate that, with our methods of collecting and processing data, about $40 \%$ of all cases of DI firing pattern were due to triggering failure. In most cases this is caused by interference with a second MUP derived from a coactive motor unit in the same muscle territory. In other words, the subjects were unable to isolate a motor unit effectively. A second mechanism of DI firing pattern seems to be a very short transient fall in the central activation of the motor unit (Fig. 4).

Our results also show that DI firing pattern (1) cannot be imitated voluntarily, (2) does not correlate with slow and irregular firing of the motor unit per se, (3) may be limited to a restricted part of motor units of the muscle, (4) is more common in the distal than in the proximal muscle, and (5) is more common in uraemic patients than in normal control subjects, while the non-uraemic neuropathy group adopts a somewhat intermediate position in this connection. Contrary to the findings of Dietz and Freund (1974, 1975), we thus propose that in most cases the occurrence of single, long firing intervals is a special feature of the central control of motor neurones, and that normal subjects and uraemic patients differ in respect to the incidence of DI firing pattern only relatively, not absolutely.

The mechanism of this firing pattern can only be speculated upon. Although the occurrence of DI firing pattern on the one hand, and the irregularity and the tendency to fatigue of motor unit firing, on the other, do not appear to correlate, they may all be signs of insufficient motor control. A hypothetical possibility still to be tested is that the sensitivity of spindle receptors drops in uraemia and results in insufficient feedback control of alphamotoneurones, thus compelling patients to use more "cortical control" to maintain the tonic activity of a single motor unit. The situa-

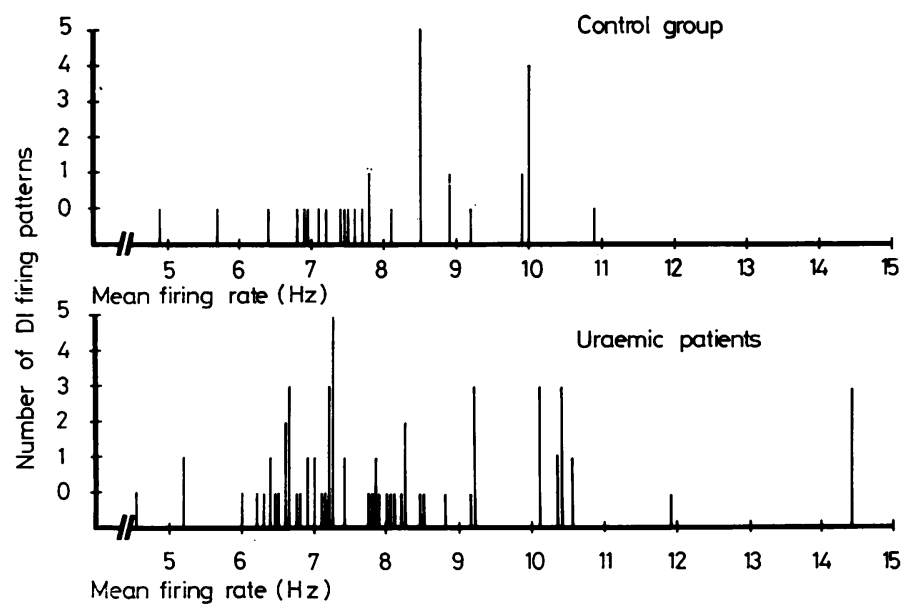

Fig. 6 Mean firing rates and number of double interval firing patterns in motor units analysed. Records made from tibialis anterior of control subjects and uraemic patients. Zero values mean no double interval firing patterns. 
tion is, thus, reminiscent of disturbed motor unit control in spastic paraplegia, where the initially tonic motor unit activity is often characteristically changed to irregular, phasic activity (Grimby et al., 1974). So far we have no experience of the occurrence of DI firing pattern in paralysed patients.

An obvious reason for the abrupt transient fall in firing frequency of a motor unit is the so-called "compensatory rest period," which occurs after a double discharge of the motor unit (Roth, 1971). We have lately shown that this phenomena is most probably caused by double firing of the motor axon. The second action potential of peripheral origin then conducts antidromically and causes inhibition of the motor neurone either by antidromic invasion to the soma, by activation of Renshaw cells or both (Halonen et al., 1977).

All these mechanisms of double interval firing dealt with above-about $80 \%$ of cases in the consecutive material analysed very carefully-do not represent the transient conduction block of the motor axons. The difference between the conclusions of Dietz and Freund $(1974,1975)$ and our own may be partly methodological. Apart from the differences in statistical analysis we did not use the single fibre EMG technique (SFEMG) as the German authors did. Our statistical methods should not be an obstacle to finding DI firing pattern. On the contrary, our method proved to be more sensitive in the sense that even one interval meeting the DI firing pattern criteria was enough to reveal the phenomenon positively. Nor does SFEMG eliminate the possibility of triggering failure, double discharge, or transient fall of central activation. The fundamental difference lies in the fact that SFEMG allows more selective study of the activity of high threshold motor units than the conventional EMG technique. According to Dietz and Freund $(1974,1975)$ double intervals occurred more frequently in high threshold units and at high repetition rate. On the other hand these findings may be partly due to the more labile central control of high threshold units. Our findings of differences in DI firing pattern incidence between the proximal and the distal muscle may be relevant in this context.

Using SFEMG, it is not possible to show conduction blocks of the main nerve fibre. Particularly if the recording is made from a single muscle fibre, a transient firing failure (supposing that trigger failures, double discharges, and central inhibition have been excluded) may originate just as well at the neuromuscular junction, nerve terminal, branching point of terminals or axon collaterals as at the main nerve fibre (Stålberg and Thiele, 1972). Despite the fact that Thiele and Stålberg (1975) were unable to show increased jitter or blocks in SFEMG of uraemic patients, these phenomena are common in many types of neuropathies, not being primarily demyelinating (Stålberg and Thiele, 1972).

A conduction block of the main nerve fibre occurs probably only when the conventional EMG record shows a synchronous firing failure of all muscle fibres of the motor unit. Positive evidence of this kind of block cannot be obtained with certainty unless a simultaneous proximal recording is made to ensure that the neurosoma has fired, but activity has not reached the periphery. This can hardly be done in man.

That a DI is due to a transient conduction block in the main motor axon can, however, be inferred with relative certainty on the basis of the following criteria: no triggering failure, no double discharge, and no transient fall in the firing frequency of a coactive MU in the vicinity of the MU under study. In only one case out of 52 DIs analysed with great accuracy were all these criteria met. We, therefore, conclude that there is not sufficient evidence that transient conduction blocking occurs in motor axons in uraemic patients.

In animal experiments it has been demonstrated that even in local experimental demyelination the safety limits for action potential conduction in nerve fibres are very high. In peripheral nerve fibres demyelinated by diphtheria toxin, transient conduction blocks did not appear unless stimulation frequency had increased up to 80-100 per second (Rasminsky and Sears, 1973). In the central nervous system the corresponding figures were even higher (McDonald and Sears, 1970).

It has been shown recently by Gilliatt and his colleagues, however, that in experiments on peripheral nerve compression in the baboon, intermittent conduction blocks occur even at firing frequencies as low as one per second (McDonald, personal communication). It would, therefore, be of interest to look for possible occurrence of DIs in demyelinating and entrapment neuropathies in man.

\section{References}

Asbury, A. K. (1975). Uremic neuropathy. In Peripheral Neuropathy. Vol. 2, pp. 982-992. Edited by P. J. Dyck, P. K. Thomas, and E. H. Lambert. W. B. Saunders: Eastbourne.

Basmajian, J. V. (1963). Control and training of individual motor units. Science, 141, 440-441.

Buchthal, F. (1973). Sensory and motor conduction in polyneuropathies. In New Developments in Electromyography and Clinical Neurophysiology. Vol. 2, 
pp. 259-271. Edited by J. E. Desmedt. Karger: Basel.

Dayan, A. D., Gardner-Thorpe, C., Down, P. F., and Gleadle, R. I. (1970). Peripheral neuropathy in uremia. Neurology (Minneapolis), 20, 649-658.

Dietz, V., and Freund, H.-J. (1974). Entladungsverhalten einzelner motorischer Einheiten bei urämischen Patienten. Ein Beitrag zur Frühdiagnose demyelinisierender Polyneuropathien. Journal of Neurology, 207, 255-269.

Dietz, V., and Freund, H.-J. (1975). Firing patterns of single motor units in the early stage of demyelinating neuropathy. Excerpta Medica, 33, 639, abstract No. 3536.

Dinn, J. J., and Crane, D. L. (1970). Schwann cell dysfunction in uraemia. Journal of Neurology, Neurosurgery and Psychiatry, 33, 605-608.

Dyck, P. J., Johnsen, W. J., Lambert, E. H., and O'Brien, P. C. (1971). Segmental demyelination secondary to axonal degeneration in uremic neuropathy. Mayo Clinic Proceedings, 46, 400-431.

Gilliatt, R. W. (1966). Nerve conduction in human and experimental neuropathies. Proceedings of the Royal Society of Medicine, 59, 989-993.

Gilliatt, R. W. (1973). Recent advances in the pathophysiology of nerve conduction. In New Developments in Electromyography and Clinical Neurophysiology. Vol. 2, pp. 2-18. Edited by J. E. Desmedt. Karger: Basel.

Grimby, L., Hannerz, J., and Rånlund, T. (1974). Disturbances in the voluntary recruitment order of anterior tibial motor units in spastic paraparesis upon fatigue. Journal of Neurology, Neurosurgery, and Psychiatry, 37, 40-46.

Halonen, J.-P., Lang, A. H., and Partanen, V. S. J. (1977). Change in motor unit firing rate after double discharge. An electromyogram study in man. Experimental Neurology, 55, 538-545.

Honet, J. C., Jebsen, R. H., Tenckhoff, H. A., and McDonald, J. R. (1966). Motor nerve conduction velocity in chronic renal insufficiency. Archives of Physical Medicine, 47, 647-652.

Jebsen, R. H., and Tenckhoff, H. A. (1969). Comparison of motor and sensory nerve conduction ve!ocity in early uremic polyneuropathy. Archives of hysical Medicine, 50, 124-126.

Kaeser, H. E. (1975). Nervous and muscular evoked potentials. In Handbook of Electroencephalography and Clinical Neurophysiology. Vol. 16A. Elsevier Scientific Publishing Company: Amsterdam.

Kemple, F. (1967). Electrodiagnosis of polyneuropathy. Electromyography, 7, 187-198.

Lang, A. H., and Forsström, J. (1977). Transient changes of sensory nerve functions in uraemia. Acta Medica Scandinavica, 202, 495-500.

McDonald, W. I., and Sears, T. A. (1970). The effects of experimental demyelination in central nervous system. Brain, 93, 583-598.

Meyer, D. W., and Lang, A. H. (1977). Does transient blocking of axonal conduction occur in uremic neuropathy? Electroencephalography and Clinical Neurophysiology, 43, 617.

Nielsen, V. K. (1973). The peripheral nerve function in chronic renal failure. V. Sensory and motor conduction velocity. Acta Medica Scandinavica, 194, 445-454.

Nielsen, V. K. (1974). The peripheral nerve function in chronic renal failure. X. Decremental nerve conduction in uremia. Acta Medica Scandinavica, 196, 83-86.

Preswick, G., and Jeremy, D. (1964). Subclinical polyneuropathy in renal insufficiency. Lancet, 2, 731732.

Rasminsky, M., and Sears, T. A. (1972). Internodal conduction in undissected demyelinated nerve fibres. Journal of Physiology (London), 227, 323-350.

Roth, G. (1971). Décharge double. Archives Suisses de Neurologie, Neurochirurgie et Psychiatrie, 108, 261272.

Stålberg, E., and Thiele, B. (1972). Transmission block in terminal nerve twigs: a single fibre electromyographic finding in man. Journal of Neurology, Neurosurgery, and Psychiatry, 35, 52-59.

Thiele, B., and Stålberg, E. (1975). Single fibre EMG findings in polyneuropathies of different aetiology. Journal of Neurology, Neurosurgery, and Psychiatry, 38, 881-887.

Thomas, P. K. (1971). Morphological basis for alterations in nerve conduction in peripheral neuropathy. Proceedings of the Royal Society of Medicine, 64, 295-298.

Thomas, P. K., Hollinrake, K., Lascelles, R. G., O'Sullivan, D. J., Baillod, R. A., Moorhead, J. F., and Mackenzie, J. C. (1971). The polyneuropathy of chronic renal failure. Brain, 94, 761-780. 